\title{
ARTICLE
}

\section{Total synthesis of palau'amine}

\author{
Kosuke Namba ${ }^{1}$, Kohei Takeuchi ${ }^{1}{ }^{2}$, Yukari Kaihara ${ }^{3}$, Masataka Oda ${ }^{4}$, Akira Nakayama $^{5}$, Atsushi Nakayama , \\ Masahiro Yoshida ${ }^{1} \&$ Keiji Tanino ${ }^{3}$
}

Palau'amine has received a great deal of attention in the past two decades as an attractive synthetic target by virtue of its intriguing molecular architecture and significant immunosuppressive activity. Here we report the total synthesis of palau'amine characterized by the construction of an ABDE tetracyclic ring core including a trans-bicylo[3.3.0]octane skeleton at a middle stage of total synthesis. The ABDE tetracyclic ring core is constructed by a cascade reaction of a cleavage of the $\mathrm{N}-\mathrm{N}$ bond, including simultaneous formation of imine, the addition of amide anion to the resulting imine (D-ring formation) and the condensation of pyrrole with methyl ester (B-ring formation) in a single step. The synthetic palau'amine is confirmed to exhibit excellent immunosuppressive activity. The present synthetic route has the potential to help elucidate a pharmacophore as well as the mechanistic details of immunosuppressive activity.

\footnotetext{
${ }^{1}$ Department of Pharmaceutical Science, Tokushima University, 1-78 Shomachi, Tokushima 770-8505, Japan. ${ }^{2}$ Graduate School of Chemical Sciences and Engineering, Hokkaido University, Sapporo 060-0810, Japan. ${ }^{3}$ Department of Chemistry, Faculty of Science, Hokkaido University, Kita-ku, Sapporo 060-0810, Japan. ${ }^{4}$ Graduate School of Medical and Dental Sciences, Niigata University, Chuo-ku, Niigata 951-8514, Japan. ${ }^{5}$ Catalysis Research Center, Hokkaido University, Sapporo 001-0021, Japan. Correspondence and requests for materials should be addressed to K.N. (email: namba@tokushima-u.ac.jp) or to K.T. (email: ktanino@sci.hokudai.ac.jp).
} 
T he pyrrole-imidazole alkaloids comprise a large family of natural products and have received a great deal of attention for their potent biological activities and tremendous structural diversity 1,2 . Palau'amine (1) was originally isolated from a sponge, Stylotella agminata, in 1993 by Scheuer and colleagues $^{3,4}$ as a novel class of pyrrole-imidazole alkaloids and the proposed structure was revised in 2007 (refs 5-9). Since the initial disclosure of a proposed structure and the later structural revision, palau'amine (1) has been an attractive synthetic target because of its intriguing molecular architecture and significant biological properties, which include antifungal, antitumour and immunosuppressive activities. The immunosuppressive activity has been of particular interest and two studies on its mode of action have been reported ${ }^{10,11}$. Thus, development of $\mathbf{1}$ as molecular probes is required for further elucidation of the potential of palau'amine as an immunosuppressive agent. In addition, investigations into the structure-activity relationship will also be needed for the development of novel lead compound of immunosuppressive agent. However, palau'amine has been a well-known natural product and proven to be the most challenging synthetic target. The noteworthy structural features of palau'amine include its two guanidine moieties, fused polycyclic system with a spiro cycle, fully substituted complex cyclopentane ring, eight contiguous stereogenic centres including a nitrogen-substituted quaternary carbon centre and trans-bicyclo[3.3.0]octane skeleton (D/E ring junction). Not surprisingly, many attempts to synthesize palau'amine, ${ }^{9}, 30$ and related compounds ${ }^{31-37}$ have been reported, and numerous reviews of these different approaches have also been published ${ }^{38-43}$. To date, however, there has been only one report of a total synthesis, by Baran and colleagues ${ }^{44}$ in 2010, which was followed by the development of an asymmetric version in 2011 (ref. 45). The most difficult challenge in the total synthesis of $\mathbf{1}$ would be the construction of a transbicyclo[3.3.0]octane system that corresponds to a D/E ring junction and Baran and colleagues ${ }^{44}$ has addressed this by adopting the transannular reaction of nine-membered lactam 2 , named macro-palau'amine, leading to $\mathbf{1}$ at the final step (Fig. 1). Despite their short and elegant synthetic route to $\mathbf{1}$, however, the total synthesis of palau'amine is still a challenging undertaking.
In particular, as the basic structure of $\mathbf{1}$ remains to be established, there is need of an efficient method for constructing an ABDE tetracyclic ring system that includes a trans-bicyclo[3.3.0]octane skeleton and, although the development of such a system will be extremely difficult, it is also absolutely critical for not only the field of synthetic organic chemistry but also the elucidation of pharmacophores and the development of palau'amine probes.

Herein we report the successful establishment of an alternative synthetic route to 1 based on the efficient construction of an ABDE tetracyclic ring core at the middle stage of total synthesis, in which many analogues of $\mathbf{1}$ possessing various ring core systems are first obtained.

\section{Results}

Synthetic plan. Our synthetic plan of $\mathbf{1}$ is outlined in Fig. 1. In this synthesis, palau'amine $\mathbf{1}$ would be obtained by the transformation of functional groups after the construction of a hexacyclic ring core 3 . The two cyclic guanidines corresponding to the $\mathrm{C}$ and $\mathrm{F}$ rings of 3 would be built on amino and carbonyl groups of $\mathrm{ABDE}$ tetracyclic ring core 4 . The $\mathrm{B}$ and $\mathrm{D}$ rings of 4 would be formed by a sequential cyclization reaction of amide and pyrrole nitrogen with the imine and methyl esters of $\mathbf{5}$, respectively. As the acylimine moiety of $\mathbf{5}$ is highly electron deficient, the nucleophilic addition of amide anion to the C10 carbon centre would occur to form a $\mathrm{D}$ ring over the steric strain of the trans-bicyclo[3.3.0] octane skeleton (D/E ring junction). The iminoester moiety of 5 would be generated from hydrazide $\mathbf{6}$ by a $\mathrm{N}-\mathrm{N}$ bond cleavage and a formation of imine at the $\mathrm{C} 10$ position. Overman et al. ${ }^{12}$ have also employed a hydrazine fragmentation in their efforts towards the palau'amine core and the reductive cleavage of the N-N bond occurred spontaneously due to the high strain of the pyrazolidine ring. On the other hand, we planned to adopt an E1cB (E-elimination, 1cB-first order with respect to conjugate base) eliminative cleavage of the N-N bond of $\mathbf{6}$, to obtain iminoester $\mathbf{5}$ directly, although, unlike in the case of reductive cleavage, there have been very few examples of $\mathrm{E} 1 \mathrm{cB}$ eliminative cleavage of $\mathrm{N}-\mathrm{N}$ bonds $\mathrm{s}^{35,46}$. The direct formation of 5 under basic conditions was expected to induce further cascade cyclization reactions leading to 4 , that is, the $\mathrm{ABDE}$ tetracyclic
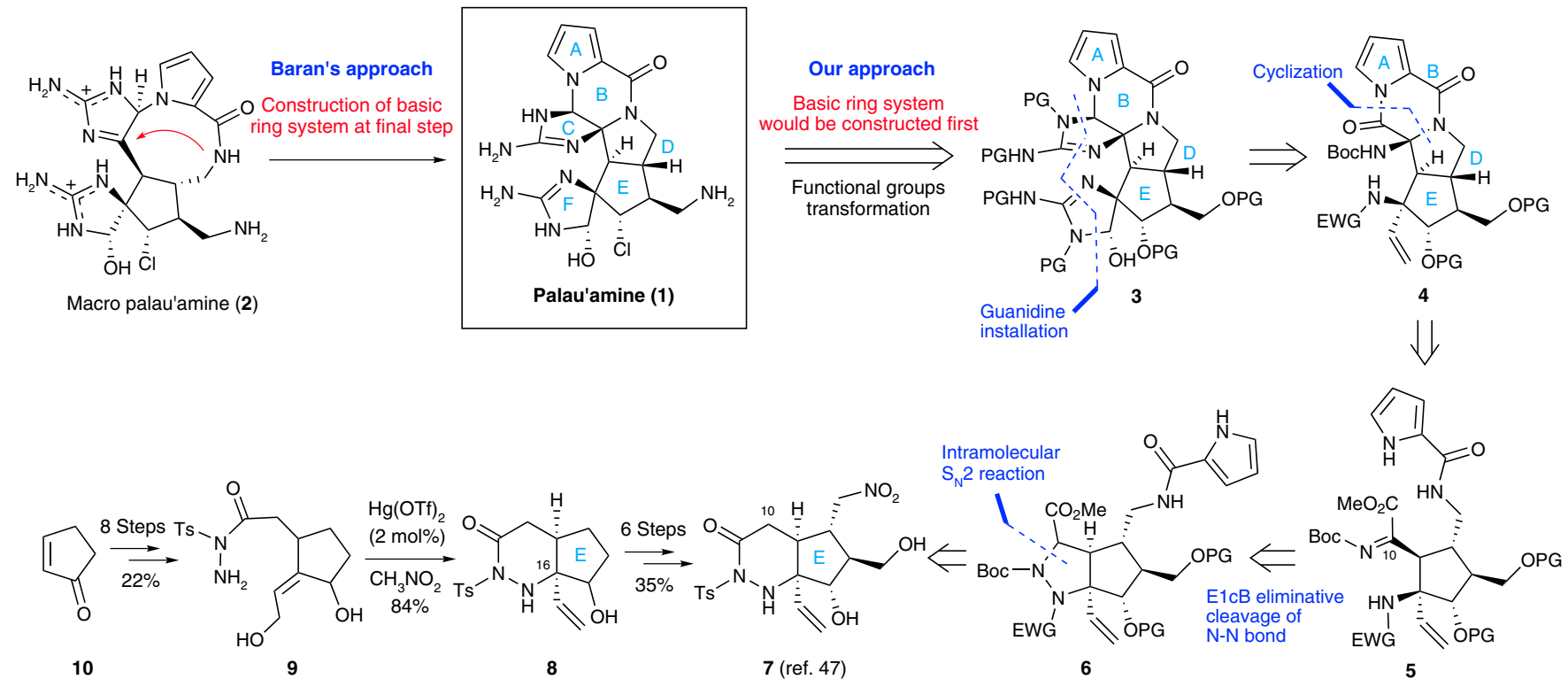

Figure 1 | Structure of palau'amine and its synthetic approach. Palau'amine 1 should be obtained by functional group transformations from the hexacyclic ring core $\mathbf{3}$, which would be constructed from the previously reported E-ring core $\mathbf{7} . \mathrm{Hg}(\mathrm{OTf})_{2}$, mercury(II) trifluomethanesulfonate; $P G$, protecting group; EWG, electron-withdrawing group. 

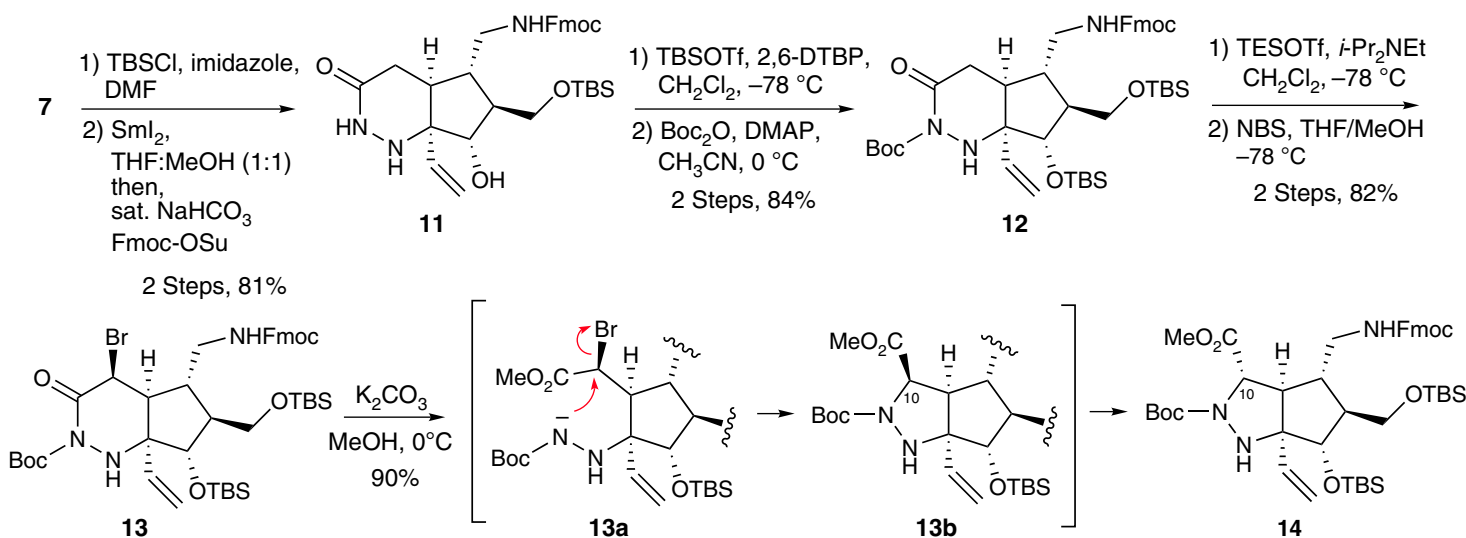

13

$13 a$

$13 b$
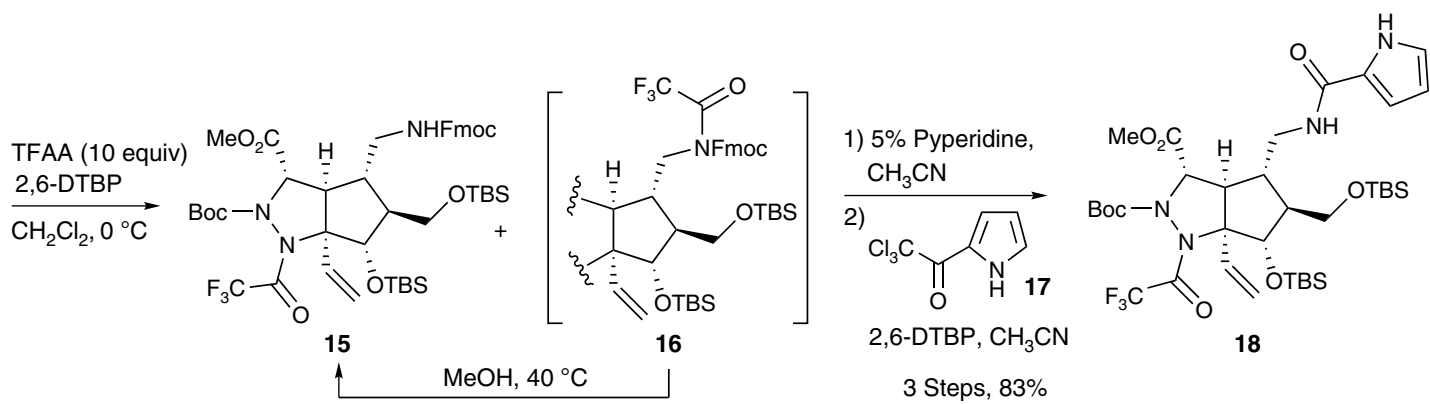

3 Steps, $83 \%$

Figure 2 | Synthesis of precursor $\mathbf{1 8}$ for the cascade reaction. The precursor $\mathbf{1 8}$ was synthesized from $\mathbf{7}$ via changes of the protecting group, bromination at the $\mathrm{C} 10$ position, metholysis followed by intramolecular $\mathrm{S}_{\mathrm{N}} 2$ reaction and introductions of trifluoroacetyl group and pyrrole amide. TBSCl, tert-butylchlorodimethylsilane; DMF, dimethylformamide; Fmoc-OSu, N-(9-fluorenylmethoxycarbonyloxy)succinimide; TBSOTf, tert-butyldimethylsilyl trifluoromethanesulfonate; 2,6-DTBP, 2,6-Di-tert-butylpyridine; $\mathrm{Boc}_{2} \mathrm{O}$, di-tert-butyl dicarbonate; DMAP, 4-dimethylaminopyridine; TESOTf, triethylsilyl trifluoromethanesulfonate; NBS, N-bromosuccinimide; TFAA, trifluoroacetic anhydride.

ring core 4 might be obtained from 6 in a single step. The pyrazolidine ring of 6 would be obtained from 7 by the introduction of an electron-withdrawing group and ring contraction via an intramolecular $\mathrm{S}_{\mathrm{N}} 2$ reaction. In 2009, we reported an efficient synthesis of the cyclopentane core 7 (ref. 47). The synthesis of 7 began with commercially available cyclopentenone 10, which was converted into acyltosylhydrazide 9 in $22 \%$ overall yield after eight steps. The mercury(II) trifluomethanesulfonate $\left(\mathrm{Hg}(\mathrm{OTf})_{2}\right)$-catalysed cyclization reaction ${ }^{48}$ of 9 proceeded smoothly to yield a tetra-substituted carbon centre corresponding to the $\mathrm{C} 16$ position of $\mathbf{1}$ and piperazine $\mathbf{8}$ was obtained in $84 \%$ yield. Compound $\mathbf{8}$ was subsequently converted into 7 as a single diastereomer in $35 \%$ yield by a sequence consisting of oxidation to enone, Morita-Baylis-Hillman reaction, 1,4-addition of nitromethane and reduction of ketone (see Supplementary Fig. 1). First, therefore, we attempted to synthesize the E1cB eliminative cleavage precursor 6 from the compound 7.

Synthesis of the cascade cyclization precursor 6 . After introduction of the tert-butylchlorodimethylsilyl (TBS) group into the primary alcohol of 7 to increase hydrophobicity, the tosyl group was reductively removed and the nitro group was reduced to the amino group by treatment with $\mathrm{SmI}_{2}$. The resulting primary amine was protected by the 9-fluorenylmethyloxycarbonyl (Fmoc) group to afford $\mathbf{1 1}$ in $81 \%$ yield from 7 . Further protections of secondary alcohol and acylhydrazide proceeded smoothly to give 12 by treatment with tert-butyldimethylsilyl trifluoromethanesulfonate as a silylation reagent followed by di-tert-butyl dicarbonate in the presence of a catalytic amount of 4-dimethylaminopyridine. As we expected, Boc protection occurred on the nitrogen possessing the acyl group due to the acidity of $\mathrm{NH}$ protons and steric hindrance ${ }^{49}$. Treatment of 12 with triethylsilyl trifluoromethanesulfonate and 2,6-di-tert-butyl pyridine at $-78^{\circ} \mathrm{C}$ readily afforded silyl ketene aminal, and the crude product was treated with $\mathrm{N}$-bromosuccinimide in a mixed solvent of tetrahydrofurane (THF) and methanol to give bromide 13 in an $82 \%$ two-step yield along with $7-14 \%$ of starting material 12 . The stereochemical outcome of $\mathbf{1 3}$ was determined by a nuclear Overhauser effect spectroscopy experiment (Supplementary Fig. 2), which indicated the $\beta$-configuration of bromide. It is likely to be that the attack of bromide on the concave face was derived from the steric hindrance of the vinyl group. Subsequent methanolysis of $\mathbf{1 3}$ afforded an amide anion 13a that immediately induced an intramolecular $\mathrm{S}_{\mathrm{N}} 2$ reaction, leading to $\mathbf{1 3 b}$. At this stage, $\mathbf{1 3 b}$ was considered to readily epimerize to 14 , to avoid the steric repulsion of a tighter concave face. The stereochemistry of 14 was determined by a nuclear Overhauser effect spectroscopy experiment (Supplementary Fig. 3). Next, we attempted to introduce a strong electron-withdrawing group to nitrogen on a tetra-substituted carbon centre. After various examinations, we found that only a trifluoroacetyl group could be introduced to afford 14 by treatment with an excess amount of trifluoroacetic anhydride. Although overacetylated product 16 was also obtained, the extra trifluoroacetyl group on the carbamate was automatically removed in methanol at $40^{\circ} \mathrm{C}$ in a quantitative yield. Finally, removal of the Fmoc group and condensation of the resulting primary amine with pyrrole trichloromethyl ketone $\mathbf{1 7}$ afforded 18 as a precursor of the key cascade reaction in an $83 \%$ three-step yield (Fig. 2).

Construction of the ABDE tetracyclic ring core. Having prepared the cleavage precursor 18, we next attempted the singlestep construction of an ABDE tetracyclic ring system. To induce an $\mathrm{E} 1 \mathrm{cB}$ eliminative cleavage of the $\mathrm{N}-\mathrm{N}$ bond, 18 was treated with 3.0 equiv of lithium hexamethyldisilazide (LHMDS) as a 


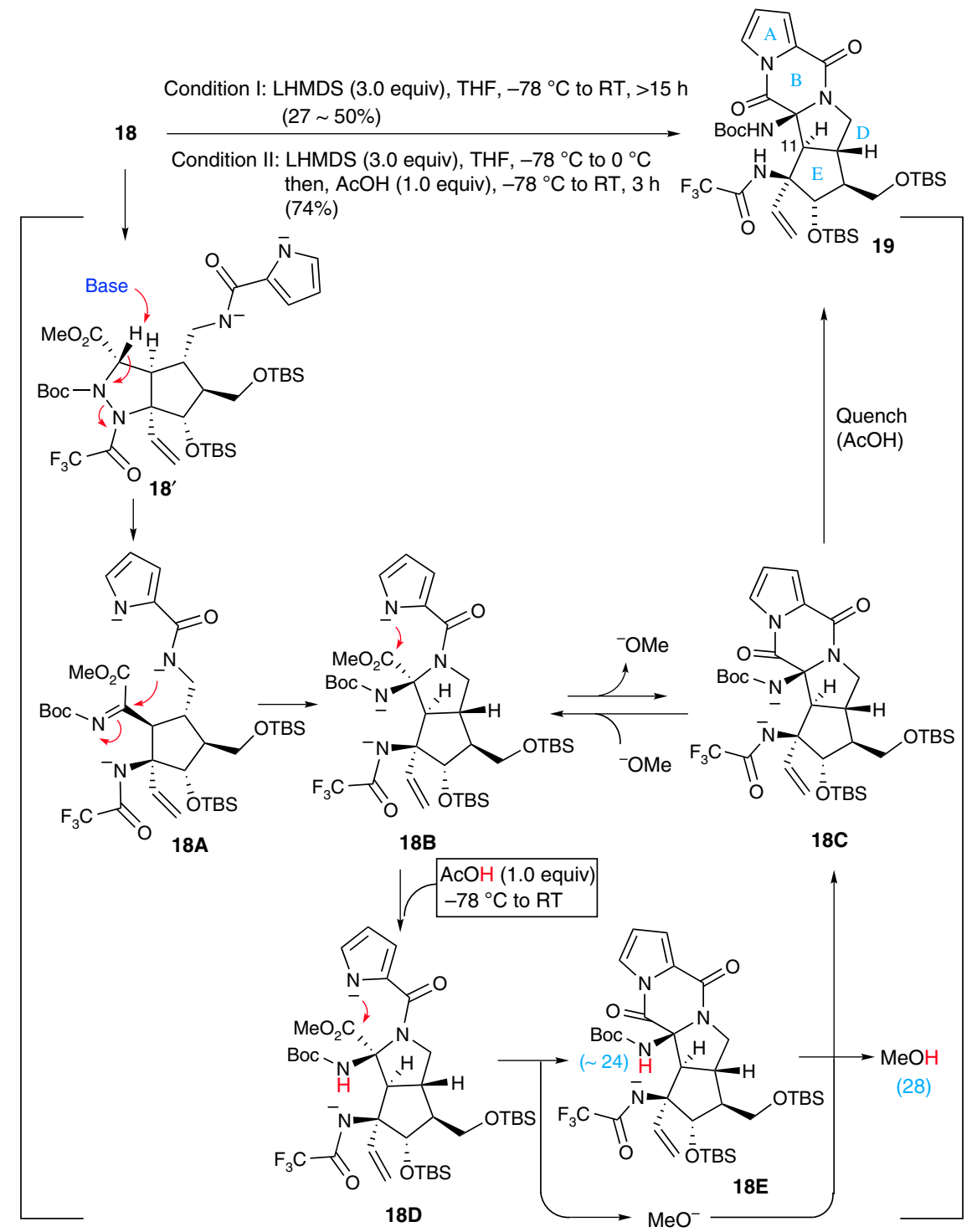

Figure 3 | Construction of ABDE ring system and comparison of the relative basicity of nitrogen anions. The ABDE tetracyclic ring core was constructed by a cascade reaction of a cleavage of the $\mathrm{N}-\mathrm{N}$ bond, including simultaneous formation of imine, the addition of amide anion to the resulting imine (D-ring formation) and the condensation of pyrrole with methyl ester (B-ring formation) in a single step. LHMDS, lithium hexamethyldisilazide.

strong base at $-78^{\circ} \mathrm{C}$ and warmed to room temperature (condition I). The reaction afforded the expected tetracyclic compound 19 that corresponds to the ABDE ring of palau'amine, including the trans-bicyclo[3.3.0] octane skeleton, in modest yield (Fig. 3). The transfused D/E ring junction and the stereochemistry of 19 were confirmed by a nuclear Overhauser effect experiment and by comparison of the coupling constant with the natural product (see Supplementary Figs 4 and 5). It was noteworthy that the coupling constant at the $\mathrm{C} 11$ proton indicated the characteristic value $(J=14.5 \mathrm{~Hz})$ of a trans-bicyclo[3.3.0] octane skeleton. The reaction pathway leading to the ABDE ring system $\mathbf{1 9}$ is explained below. Treatment of $\mathbf{1 8}$ with 3.0 equiv of a strong base would abstract two $\mathrm{NH}$ protons and hydrogen at the $\mathrm{C} 10$ position, thereby inducing a $\beta$-elimination of nitrogen possessing an electron-withdrawing group of 18', as shown in Fig. 3. This would lead simultaneously to both the cleavage of the N-N bond and the imine formation at the $\mathrm{C} 10$ position to give 18A. The nucleophilic addition of amide anion to the electron-deficient C10 carbon centre would occur immediately to give $\mathbf{1 8 B}$ possessing a trans-bicyclo[3.3.0]octane skeleton (D/E ring junction). Furthermore, the cascade reaction would not stop at 18B due to the remaining pyrrole anion and subsequent condensation of pyrrole with methyl ester formed a B ring to give $\mathbf{1 9}$ (Fig. 3). The results encouraged us to scale up this reaction from a few milligrams to $100 \mathrm{mg}$ with the goal of completing the total synthesis of palau'amine. However, the reaction was found to suffer from poor reproducibility, often leading to poor to low yields even after a prolonged reaction time. After careful consideration of the reaction mechanism, we established an improved method involving the partial protonation of the anionic intermediates with acetic acid (condition II). On treatment with 3.0 equiv of LHMDS, substrate 18 undergoes stepwise lithiation of the two $\mathrm{NH}$ protons at $-78^{\circ} \mathrm{C}$ and then the $\mathrm{C} 10$-proton at around $0{ }^{\circ} \mathrm{C}$. Once the $\mathrm{C} 10$ proton is abstracted, the formation of $\mathbf{1 8 A}$ and the subsequent cyclization of amide anion leading to 18B would proceed rapidly and completely. Indeed, only $18 \mathrm{~B}$ was detected as its protonated form by thin layer chromatography (TLC) (see Supplementary Fig. 6), indicating the fast conversion of $18 \mathrm{~A}$ to $18 \mathrm{~B}$ and the slow formation of $18 \mathrm{C}$ from $18 \mathrm{~B}$. The smaller $\mathrm{pKa}$ value (in dimethyl sulfoxide) of the pyrrole moiety 
a

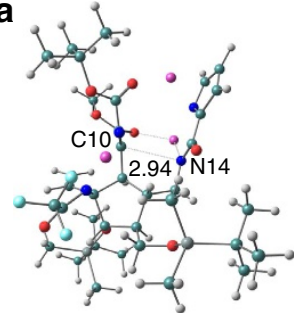

18A

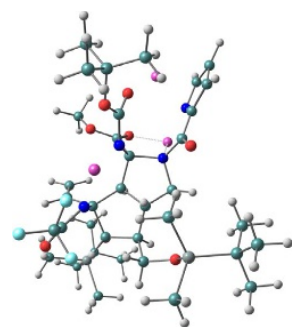

18B

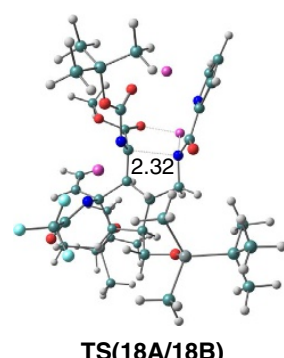

TS(18A/18B)

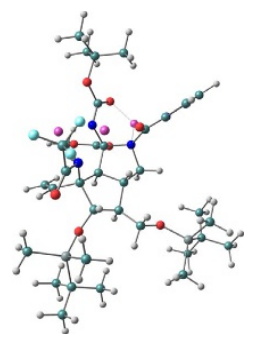

$18 \mathrm{~B}^{\prime}$ b

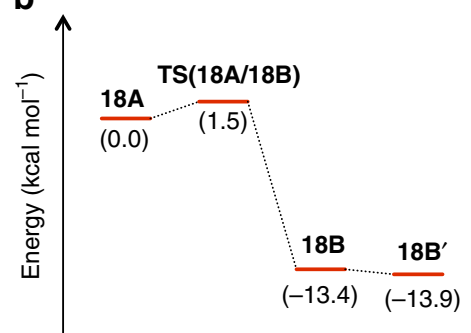

Figure 4 | Optimized structures and energy diagram of 18. (a) Optimized structures of 18A, 18B, TS(18A/18B) and 18B'. TS(18A/18B) is a transition-state structure connecting $\mathbf{1 8 A}$ and $\mathbf{1 8 B}$. 18B' represents a structure after the translocation of a lithium atom. The atoms were colour coded as follows: grey, H; pink, Li; green, C; blue, N; red, O; light blue, F; dark grey, Si. (b) Potential energy profile for the cyclization reaction (18A $\rightarrow \mathbf{1 8 B}$ ). Calculations were performed by the density functional theory (DFT) using the modern functionals of M06-2X with the 6-31G* basis set. The solvent effects are taken into account by the self-consistent reaction field (THF). The potential energies (in $\mathrm{kcal} \mathrm{mol}^{-1}$ ) relative to 18A are shown in parentheses. All calculations were performed by the Gaussian 09 package ${ }^{58}$.

(should be $<23$ of pyrrole) compared with that of methanol (28) predicted that the equilibrium between $18 \mathrm{~B}$ and $\mathbf{1 8 C}$ favoured the former, which led us to remove the methoxide ion without quenching the pyrrole anion. Thus, after checking the conversion of 18 into $18 \mathrm{~B}$ by TLC, the mixture was cooled to $-78^{\circ} \mathrm{C}$ again and an exact 1.0 equiv of acetic acid was slowly added. On warming to room temperature, the desired product 19 was obtained in up to $74 \%$ yield with good reproducibility in acceptable scales for total synthesis (Fig. 3).

The mechanism underlying the cascade reaction under 'condition II' is explained as follows. The intermediate 18B possesses three nitrogen anions of Boc-carbamate $(\sim 24)$, pyrrole $(<23)$ and trifluoroacetoamide $(\sim 17)$, according to the order of basicity by comparison of the $\mathrm{pKa}$ values of protonated $\mathrm{NH}$ functional groups. Thus, an exact 1.0 equiv of acetic acid would protonate only the anion of Boc-carbamate and trianion 18B would be converted into dianion 18D. Although the mixture was warming to room temperature, the remaining pyrrole anion induced condensation with methyl ester, simultaneously generating methoxide as in the case with trianion. However, as the condensation product $18 \mathrm{E}$ possesses an active $\mathrm{NH}$ proton, unlike in the case with trianion, methoxide does not attack the pyrrole amide but extracts the active Boc-NH proton to give the same dianion $18 \mathrm{C}$ with a quenching methoxide anion. Among various acids, including phenol and imide derivatives, acetic acid was found to be the best protonating reagent. In addition, precise amounts of LHMDS and acetic acid are very important for this $\mathrm{pKa}$ game reaction. As stated above, we succeeded in establishing an efficient method for constructing the basic core structure of palau'amine.

Theoretical calculations on the coordination effect of lithium. The remaining question in this cascade reaction is how the amide anion of 18A got close to the $\mathrm{C} 10$ carbon centre, to overcome the steric strain of the trans-bicyclo[3.3.0]octane skeleton, although the Boc-imine moiety of $\mathbf{1 8 A}$ was highly electron deficient. Thus, we focused on the coordination effect of lithium ion. If the lithium countercation of amide anion formed a coordination bond with the carbonyl group of methyl ester, the amide nitrogen (N14) and the C10 carbon centre would be located at transannular positions that are close to each other due to the strain of the eight-membered ring. To investigate this coordination effect, theoretical calculations were carried out by the density functional theory method. The optimized structure of $\mathbf{1 8 A}$ including lithium ions is shown in Fig. 4a (the effects of coordination of solvent THF molecules to lithium ions are discussed in the Supplementary Data; see the Supplementary Discussion and Supplementary Figs 7 and 8). Interestingly, the calculation actually indicated not only the expected coordination of lithium amide to the carbonyl group of methyl ester but also the unpredictable coordination of lithium salt of pyrrole anion to the carbonyl oxygen of the Boc group. Therefore, the two trans-oriented side chains were quite close to each other by the chelation to two lithium ions and the distance between N14 and C10 at the transannular positions was calculated to be only $2.94 \AA$. Owing to the short distance between the two reaction points, the energy barrier of the cyclization reaction $(\mathbf{1 8 A} \rightarrow \mathbf{1 8 B})$ was estimated to be only $1.5 \mathrm{kcal} \mathrm{mol}^{-1}$, allowing the reaction to proceed smoothly (see Fig. $4 \mathrm{~b}$ for the potential energy profile of the cyclization reaction). Thus, the nucleophilic addition of the amide anion (N14) occurred before the rotation of the single bond of $\mathrm{C} 10-\mathrm{C} 11$, which was also restricted by the coordination effect of lithium ion, to afford 19 as a single diastereomer possessing the $\beta$-configuration of the NHBoc group at the $\mathrm{C} 10$ position. From the calculated result, it was concluded that the chelation effect of lithium ion played a significant role in the formation of the trans-bicyclo[3.3.0]octane skeleton. In fact, this cascade reaction in the presence of 3.6 equiv of hexamethylphosphoric triamide as a lithium ion scavenger afforded a complex mixture and the desired cyclized (D/E ring) products and related intermediates were not detected. Furthermore, the use of other bases, such as sodium hexamethyldisilazide (NaHMDS) and potassium hexamethyldisilazide (KHMDS), also did not give any 


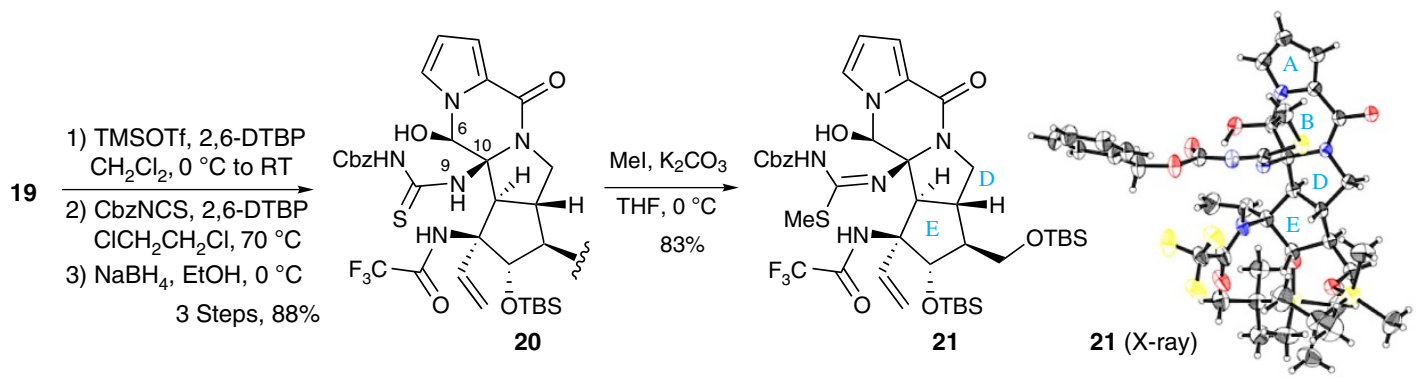

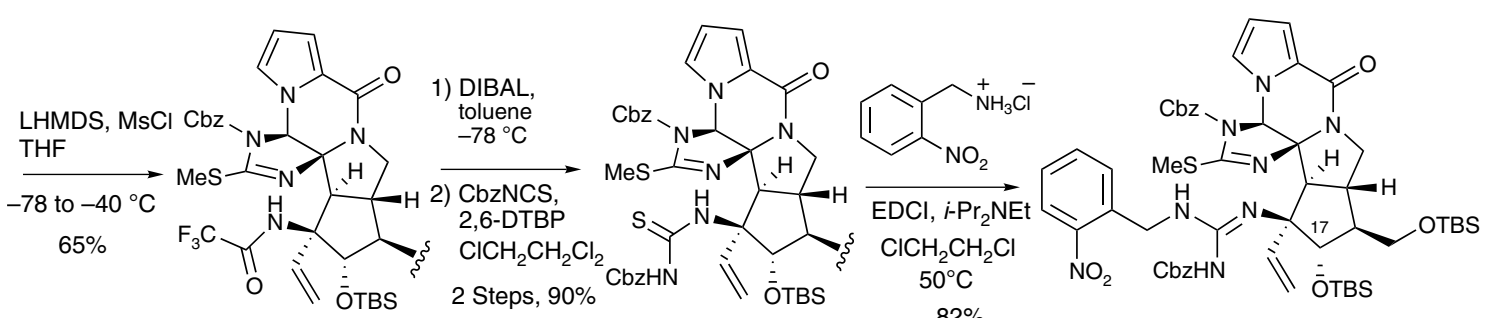

22

1) HF•Pyr, THF

2) TIPSOTf, 2,6-lutidine, $\mathrm{CH}_{2} \mathrm{Cl}_{2}, 0^{\circ} \mathrm{C}$ to $\mathrm{RT}$

2 Steps, $67 \%$

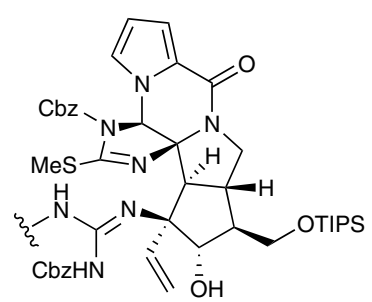

25
23

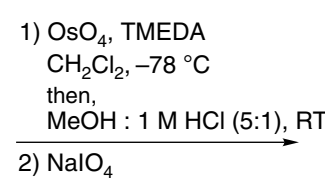

1) $\mathrm{OsO}_{4}, \mathrm{TMEDA}$

2) $\mathrm{NalO}$ $\mathrm{MeOH}: \mathrm{H}_{2} \mathrm{O}(4: 1), \mathrm{RT}$

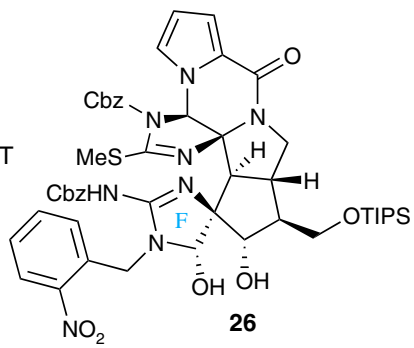

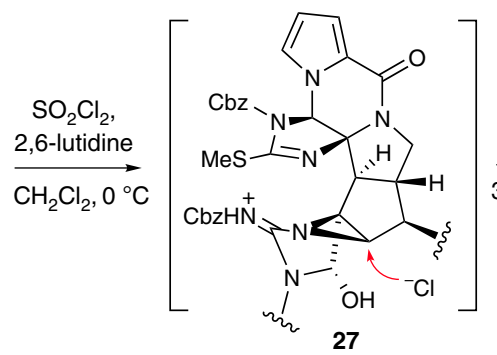

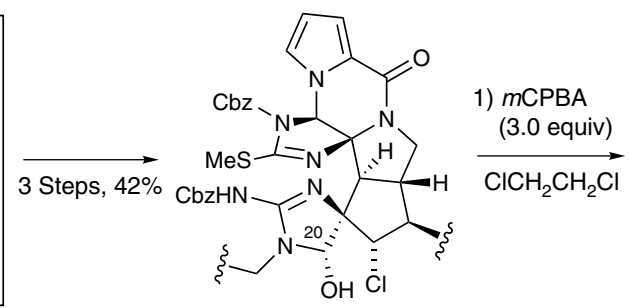

28

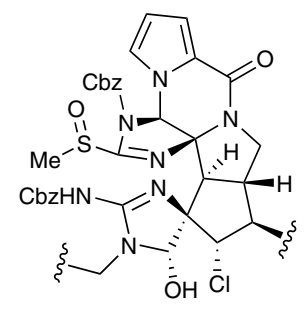

29

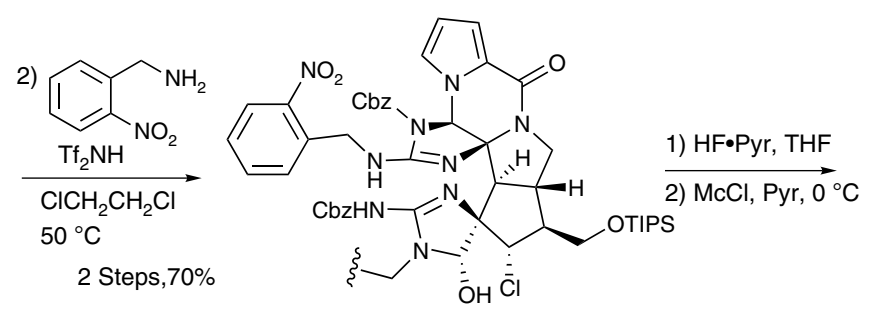

30

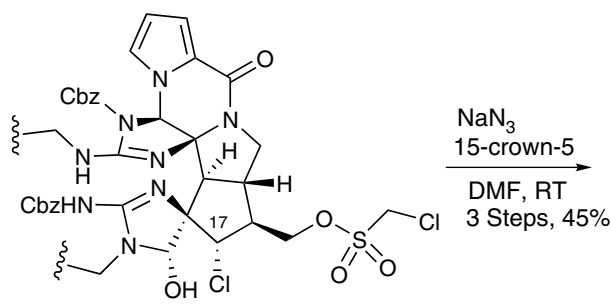

31

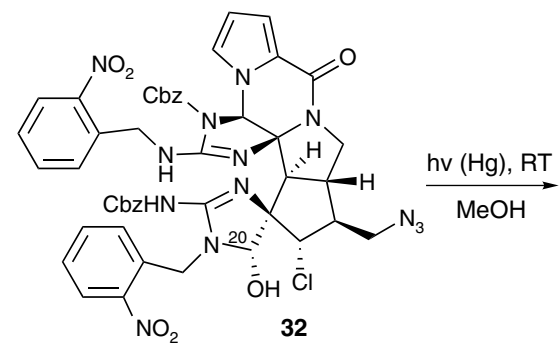

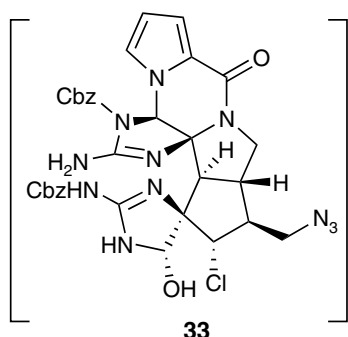

Figure $\mathbf{5}$ | Total synthesis of palau'amine 1. The ABDE tetracyclic ring core $\mathbf{1 9}$ was successfully converted into $\mathbf{1}$ by the sequential operations of a $C$-ring formation, a F-ring formation, a substitution of chloride at C17 for secondary alcohol, a guanidine formation on the C-ring, substitution of an amine equivalent for the primaly alcohol and deprotections. CbzNCS, N-benzyloxycarbonyl isothiocyanate; MsCl, methanesulfonyl chloride; DIBAL, diisobutylaluminium hydride; EDCI, 1-ethyl-3-(3-dimethylaminopropyl)carbodiimide; TIPSOTf, triisopropylsilyl trifluoromethanesulfonate; TMEDA, tetramethylethylenediamine; $m C P B A$, m-chloroperoxybenzoic acid; $\mathrm{McCl}$, chloromethylsulfonyl chloride. 


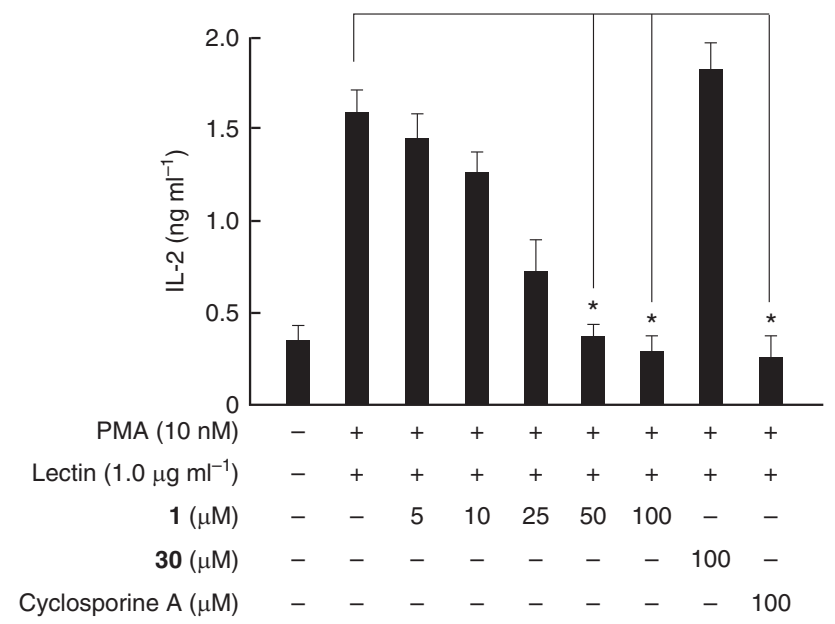

Figure 6 | Immunosuppressive activity of synthetic palau'amine at various concentrations. Balb/c splenic lymphocytes were treated with various concentrations of an aqueous solution of synthetic $\mathbf{1}$, synthetic $\mathbf{3 0}$ $(100 \mu \mathrm{M})$ and cyclosporine $\mathrm{A}(100 \mu \mathrm{M})$ at $37^{\circ} \mathrm{C}$ for $1 \mathrm{~h}$, and then the cells were incubated with phorbol 12-myristate 13 -acetate (PMA, $10 \mathrm{nM}$ ) and lectin $\left(1.0 \mu \mathrm{g} \mathrm{ml}^{-1}\right)$. After incubation for $5 \mathrm{~h}$, the IL-2 in culture supernatant was measured by using an enzyme-linked immunosorbent assay kit (error bars, s.e.m.; ${ }^{\star} P<0.01$ ).

desired cyclization products. In addition, the yield of tetracyclic 19 was dramatically decreased to $10 \sim 20 \%$, when the initial treatment of 3.0 equiv of LHMDS was directly conducted at $0^{\circ} \mathrm{C}$. This result clearly suggested that the coordination of lithium ions to the two carbonyl groups must be formed first at $-78^{\circ} \mathrm{C}$ before the extraction of the $\mathrm{C} 10$ proton occurs at $0^{\circ} \mathrm{C}$.

Total synthesis of palau'amine. Having established an efficient method for constructing an $\mathrm{ABDE}$ ring system that overcame the most difficult barrier to the total synthesis of palau'amine, we attempted a total synthesis from 19 (Fig. 5). We first tried to construct a C-ring by using an amino group (N9) on C10 and a carbonyl group at $\mathrm{C} 6$ as a foothold. The amino group (N9) of 19 was converted into thiourea by removal of the Boc group according to Ohfune's method ${ }^{50}$ followed by treatment with $\mathrm{N}$-benzyloxycarbonyl isothiocyanate (CbzNCS) and the pyrrole amide at C6 was selectively reduced to hemiaminal $\mathbf{2 0}$ as an isolable compound in $88 \%$ overall yield after three steps from 19 . The removal of the Boc group did not proceed after the reduction of the pyrrole amide, indicating that the planar configuration of the $s p^{2}$ carbon centre reduced the steric hindrance around the N9 amino group. Furthermore, the use of carbodiimide derivatives for the direct formation of guanidine did not proceed and only CbzNCS as a small and reactive reagent reacted with the sterically hindered resulting N9 amine. As the thiourea moiety of $\mathbf{2 0}$ could not be directly converted into guanidine due to the predominant cyclization from hemiaminal oxygen to activated thiourea, the thiourea moiety was converted into isothiourea $\mathbf{2 1}$. At this stage, the structure of the synthetic intermediate including the trans-bicyclo[3.3.0]octane skeleton was unambiguously confirmed by an X-ray diffraction study (Supplementary Method and Supplementary Data 1). Treatment of 21 with LHMDS and methanesulfonyl chloride at -78 to $-40{ }^{\circ} \mathrm{C}$ induced a sequential reaction of mesylation, the elimination of mesylate and the addition of nitrogen to the isothiourea moiety to give pentacyclic 22 in $65 \%$ yield, in a manner similar to that of the synthesis of $(+)$-dibromophakellstatin reported by Nagasawa and colleagues ${ }^{51}$. Having constructed a C-ring, we next tried to form an F-ring. Only the reductive condition using diisobutylaluminium hydride was successful for the removal of the trifluoroacetyl group and subsequent treatment of the resulting amine with CbzNCS afforded thiourea $\mathbf{2 3}$ in good yield. The thiourea moiety was directly converted into guanidine 24 by the condensation using 1-ethyl-3-(3-dimethylaminopropyl)carbodiimide ${ }^{52}$ with $o$-nitrobenzylamine in $82 \%$ yield. However, the oxidative cleavage of the vinyl group without the oxidation of pyrrole was difficult, probably due to the steric hindrance of the TBS group at the $\mathrm{C} 17$ position. Thus, two TBS groups were removed by hydrogen fluoride (HF) · pyridine and only primary alcohol was protected again by the triisopropylsilyl group, to give 25 in a $67 \%$ two-step yield. In the case of the free secondary hydroxyl group, dihydroxylation using $\mathrm{OsO}_{4}$ and tetramethylethylenediamine in dichloromethan $e^{53}$ proceeded smoothly at $-78^{\circ} \mathrm{C}$ to give osmate and subsequent hydrolysis in the mixed solution of methanol and $1 \mathrm{M} \mathrm{HCl}$ afforded the desired diol. The oxidative cleavage of diol was successful only in the mixed solution of methanol and water (4:1) to give an unstable F-ring product 26, which gradually decomposed even under the neutral condition, due probably to the retro-aldol reaction at the $\mathrm{C} 17$ position. Product 26 was then directly used for the next reaction without purification. With the construction of an ABCDEF ring system of palau'amine, the conversions of functional groups into $\mathbf{1}$ were finally attempted. The substitution of the secondary hydroxyl group to chloride with stereoretention proceeded by the use of the neighbouring-group effect of guanidine on the F-ring. The activation of secondary alcohol by using sulfuryl chloride induced the participation of guanidine depicted as $\mathbf{2 7}$ in analogy with 'massadine aziridine 54 and the subsequent nucleophilic attack of chloride afforded 28 with an $\alpha$-configuration of chloride, along with a small amount of epimer at the C20 position as an unnatural configuration. On the other hand, the hydroxyl group of hemiaminal also reacted with excess sulfuryl chloride to give dichloride when more than 1.0 equiv of sulfuryl chloride was used, and the chloride at the C20 position was readily hydrolysed to convert back to 28 . Next, we had to develop a new method for converting the methylthio group into an amino group, leading to guanidine under acidic conditions, because we found that hexacyclic intermediates were readily decomposed under the basic conditions. After various examinations, we achieved the desired conversion by the reaction of a trifluoromethanesulfonyl imide salt of $o$-nitrobenzylamine with sulfoxide 29 to give $\mathbf{3 0}$ in a $70 \%$ two-step yield. The overoxidation into sulfone and the use of a salt other than trifluoromethanesulfonic imide afforded only a complex mixture. In the final transformation of the functional group, the primary alcohol was converted into chloromethanesulfonate 31 (ref. 55) after removal of the triisopropylsilyl group. The azidation reaction of $\mathbf{3 1}$ proceeded at room temperature to afford $\mathbf{3 2}$ as a protected palau'amine. When methanesulfonate was adopted as a leaving group, the azidation reaction required a high temperature $\left(50^{\circ} \mathrm{C}\right)$ that also induced azidation at the $\mathrm{C} 17$ position via the neighbouring-group effect. Fortunately, the minor epimer of the unnatural configuration at the C20 position disappeared at this stage. Finally, Hg-lump irradiation followed by direct hydrogenation as a deprotection of $\mathbf{3 2}$ afforded $\mathbf{1}$ in $64 \%$ yield as a 3TFA salt. Synthetic 1 showed spectral data $\left({ }^{1} \mathrm{H}\right.$ and ${ }^{13} \mathrm{C}$ nuclear magnetic resonance (NMR), high-resolution mass spectra) completely identical to those of natural palau'amine $\mathbf{1}$ (refs 3,7 ). Throughout this total synthesis, 1 was obtained in $0.039 \%$ overall yield after 45 steps (78\% average yield at each step) from commercially available cyclopentenenone 10. As a result of the 45-step synthesis, many synthetic intermediates as derivatives of $\mathbf{1}$ possessing various ring core systems were actually obtained for the first time. In future studies, we plan to perform activity evaluations for each deprotected synthetic intermediate, to further elucidate the pharmacophore. 
Biological test of synthetic palau'amine. Finally, the immunosuppressive activity of synthetic palau'amine was examined (Fig. 6). Lymphocytes derived from a mouse spleen were treated with various concentrations of an aqueous solution of synthetic 1 for $1 \mathrm{~h}$ and then the cells were incubated with phorbol 12 -myristate 13 -acetate and lectin ${ }^{56}$. After incubation for $5 \mathrm{~h}$, the interleukin-2 (IL-2) in the culture supernatant was measured by using an enzyme-linked immunosorbent assay kit. Thereupon, the synthetic palau'amine 3 TFA salt was confirmed to exhibit strong immunosuppressive activity, as shown in Fig. 6. The IC50 value was determined to be $45.3 \mu \mathrm{M}$, which is a practical level of activity, as it was for cyclosporine A. Not surprisingly, the synthetic intermediate $\mathbf{3 0}$ as a negative control did not exhibit immunosuppressive activities, due to the large hydrophobic protecting groups of all polar functional groups, and it slightly increased the production of IL-2. Therefore, we confirmed that not only the spectral data but also the biological properties of synthetic 1 were identical to those of natural palau'amine. Recently, Tepe and coworkers ${ }^{10,11}$ reported that palau'amine $\mathbf{1}$ and its related natural products inhibit $\mathrm{I} \kappa \mathrm{B} \alpha$ degradation. On the other hand, we revealed that the synthetic palau'amine•3TFA salt inhibited the release of IL-2 from lymphocytes in the same manner as cyclosporine $\mathrm{A}$, which is known to inhibit the $\mathrm{I} \kappa \mathrm{B} \alpha$ degradation and nuclear factor $-\kappa \mathrm{B}^{57}$. Thus, our synthetic palau'amine 3 TFA salt was also expected to exhibit a similar inhibition of $\mathrm{I} \kappa \mathrm{B} \alpha$ degradation.

\section{Discussion}

In summary, we have achieved the total synthesis of palau'amine 1 via the single-step construction of an ABDE tetracyclic ring system. In the single-step construction, the chelation effect of lithium salt forming an eight-membered ring was crucial for the construction of a trans-bicyclo[3.3.0] octane skeleton (D/E ring junction) by bringing two reaction points close to each other at the transannular position. With the establishment of an efficient method for constructing a tetracyclic basic structure of palau'amine, various synthetic analogues for structure-activity relationship study and chemical probes of palau'amine would be accessible. For example, protected palau'amine $\mathbf{2 8}$ might be able to introduce labeling groups at desired spots on a C-ring, F-ring or a primary amine of an E-ring. Therefore, the chemistry described here offers not only a solution to a formidable synthetic challenge but also an alternative synthetic route to elucidate a pharmacophore and the mechanistic details of bioactivities of palau'amine. As our synthetic route is actually too long to develop $\mathbf{1}$ as a practical immunosuppressive agent at the current stage, further improvement of the key cascade cyclization reaction and the development of a shorter route to the cyclization precursors as a second-generation synthesis are also currently underway in our laboratory.

\section{Methods}

General. All the reaction were carried out in a round-bottomed flask with an appropriate number of necks and side arms connected to a three-way stopcock and/or a rubber septum cap under an argon atmosphere. All vessels were first evacuated by rotary pump and then flushed with argon before use. Solution and solvent were introduced by hypodermic syringe through a rubber septum. During the reaction, the vessel was kept under a positive pressure of argon. Dry THF was freshly prepared by distillation from benzophenone ketyl before use. Anhydrous $\mathrm{CH}_{2} \mathrm{Cl}_{2}$, dimethylformamide, ethanol, $\mathrm{MeCN}$, methanol, pyridine and toluene were purchased from Kanto Chemical Co. Inc.

Infrared spectra were recorded on JASCO FT/IR-4100 spectrophotometer using $5 \mathrm{~mm} \mathrm{KBr}$ plate. Wavelengths of maximum absorbance are quoted in $\mathrm{cm}^{-1}$.

${ }^{1} \mathrm{H}-\mathrm{NMR}$ spectra were recorded on a JEOL ECA-400 (400 MHz), JEOL ECA-500 $(500 \mathrm{MHz})$ and Bruker AV-500 $(500 \mathrm{MHz})$ in $\mathrm{CDCl}_{3}, d-\mathrm{MeCN}$ and $\mathrm{D}_{2} \mathrm{O}$, respectively. Chemical shifts are reported in p.p.m. and signals are expressed as singlet $(\mathrm{s})$, doublet $(\mathrm{d})$, triplet $(\mathrm{t})$, quartet $(\mathrm{q})$, multiplet $(\mathrm{m})$ and broad (br).

${ }^{13} \mathrm{C}-\mathrm{NMR}$ spectra were recorded on a JEOL ECA-400 (100 MHz), Bruker AV-400N
$(100 \mathrm{MHz})$ and Bruker AV-500 (125 MHz) in $\mathrm{CDCl}_{3}, \mathrm{C}_{6} \mathrm{D}_{6}, \mathrm{CD}_{3} \mathrm{CN}$ and $\mathrm{D}_{2} \mathrm{O}$. Chemical shifts are reported in p.p.m. High-resolution mass spectra were recorded on a Thermo Scientific Exactive, Instrumental Analysis Division, Equipment Manager Center Creative Research Institution, Hokkaido University and a Waters SYNAPT-G2 Si HDMS, Tokushima Bunri University. HPLC was recorded on a HITACHI D-2,500 Chromato-Integrater. Analytical TLC was performed using $0.25 \mathrm{~mm}$ E Merck Silica gel (60F-254) plates. Reaction components were visualized with phosphomolybdic acid or ninhydrin or $p$-anisaldehyde in $10 \%$ sulfuric acid in ethanol. Kanto Chem. Co. Silica Gel 60N (particle size $0.040-0.050 \mathrm{~mm}$ ) was used for column chromatography. Mouse IL-2 ELISA was purchased from Biolegend.

Experimental data. For ${ }^{1} \mathrm{H}$ and ${ }^{13} \mathrm{C}$ NMR spectra of compounds, see Supplementary Figs 9-48. For the comparisons of ${ }^{1} \mathrm{H}$ and ${ }^{13} \mathrm{C}$ spectra of the natural and synthetic palau'amine, see Supplementary Figs 49 and 50, and Supplementary Tables 1 and 2. For the HPLC of synthetic palau'amine, see Supplementary Fig. 51 For the Cartesian Coordinates from density functional theory calculations (in $\AA$ ) of 18, see Supplementary Tables 3-7. For the experimental procedures and spectroscopic and physical data of compounds and the crystallographic data of compound 21, see Supplementary Methods.

\section{References}

1. Forte, B. et al. A submarine journey: the pyrrole-imidazole alkaloids. Mar. Drugs 7, 705-753 (2009).

2. Mourabit, A. A. 1. \& Potier, P. Sponge's molecular diversity through the ambivalent reactivity of 2-aminoiidazole: a universal chemical pathway to the oroidin-based pyrrole-imidazole alkaloids and their palau'amine congeners. Eur. J. Org. Chem. 2001, 237-243 (2001)

3. Kinnel, R. B., Gehrken, H.-P. \& Scheuer, P. J. Palau'amine: a cytotoxic and immunosuppressive hexacyclic bisguanidine antibiotic from the sponge Stylotella agminata. J. Am. Chem. Soc. 115, 3376-3377 (1993).

4. Kinnel, R. B., Gehrken, H.-P., Swali, R., Skoropowski, G. \& Scheuer, P. J. Palau'amine and its congeners: a family of bioactive bisguanidines from the marine sponge Stylotella aurantium. J. Org. Chem. 63, 3281-3286 (1998).

5. Grube, A. \& Köck, M. Structural assignment of tetrabromostyloguanidine: does the relative configuration of the palau'amines need revision. Angew. Chem. Int. Ed. 46, 2320-2324 (2007).

6. Buchanan, M. S. et al. Natural products, stylissadines A and B, specific antagonists of the $\mathrm{P}_{2} \mathrm{X}_{7}$ receptor, an important inflammatory target. J. Org. Chem. 72, 2309-2317 (2007).

7. Buchanan, M. S., Carroll, A. R. \& Quinn, R. J. Revised structure of palau'amine. Tetrahedron Lett. 48, 4573-4574 (2007).

8. Kobayashi, H. et al. Carteramine a, an inhibitor of neutrophil chemotaxis, from the marine sponge stylissa carteri. Tetrahedron Lett. 48, 2127-2129 (2007).

9. Lanman, B. A., Overman, L. E., Paulini, R. \& White, N. S. On the structure of palau'amine: evidence for the revised relative configuration from chemical synthesis. J. Am. Chem. Soc. 129, 12896-12900 (2007).

10. Lansdell, T. et al. Palau'amine and related oroidin alkaloids dibromophakellin and dibromophakellstatin inhibit the human 20 S proteasome. J. Nat. Prod. 75, 980-985 (2012).

11. Beck, P., Lansdell, T. A., Hewlett, N. M., Tepe, J. J. \& Groll, M. Indolophakellins as $\beta 5$-specific noncovalent proteasome inhibitors. Angew. Chem. Int. Ed. 54, 2830-2833 (2015).

12. Overman, L. E., Rogers, B. N., Tellew, J. E. \& Trenkle, W. C. Stereocontrolled synthesis of the tetracyclic core of the bisguanidine alkaloids palau'amine and styloguanidine. J. Am. Chem. Soc. 119, 7159-7160 (1997).

13. Dilley, A. S. \& Romo, D. Enantioselective strategy to the spirocyclic core of palau'amine and related bisguanidine marine alkaloids. Org. Lett. 3, 1535-1538 (2001).

14. Belanger, G. et al. Stereocontrolled synthesis of triazacyclopenta $[c d]$ pentalenes by intramolecular 1,3-dipolar cycloaddition reactions of azomethine imines. J. Org. Chem. 67, 7880-7883 (2002).

15. Poullennec, K. G., Kelly, A. T. \& Romo, D. Highly diastereoselective desymmetrizations of cyclo(pro,pro): an enantioselective strategy toward phakellstatin and phakellin. Org. Lett. 4, 2645-2648 (2002).

16. Poullennec, K. G. \& Romo, D. Enantioselective total synthesis of (+)-dibromophakellstatin. J. Am. Chem. Soc. 125, 6344-6345 (2003).

17. Katz, J. D. \& Overman, L. E. Studies towards the total synthesis of palau'amine. Formation of 4,5-dihydropyrrole-2-carboxylate intermediates by alkeneenamide ring-closing metathesis. Tetrahedron 60, 9559-9568 (2004).

18. Garrido-Hernandez, H. et al. Spirocycloisomerization of tethered alkylidene glycocyamidines: synthesis of a base template common to the palau'amine family of alkaloids. Angew. Chem. Int. Ed. 44, 765-769 (2005).

19. Dransield, P. J., Dilley, A. S., Wang, S. \& Romo, D. A unified synthetic strategy toward oroidin-derived alkaloids premised on a biosynthetic proposal. Tetrahedron 62, 5223-5247 (2006).

20. Lanman, B. A. \& Overman, L. E. Evaluation of strategies for the synthesis of the guanidine hemiaminal portion of palau'amine. Heterocycles $\mathbf{7 0 , 5 5 7 - 5 7 0}$ (2006). 
21. Nakadai, M. \& Harran, P. G. Concerning the phakellin substructure of palau'amine. Tetrahedron Lett. 47, 3933-3935 (2006).

22. Bultman, M. S., Ma, J. \& Gin, D. Y. Synthetic access to the chlorocyclopentane cores of the proposed original and revised structures of palau'amine. Angew. Chem. Int. Ed. 47, 6821-6824 (2008).

23. Cernak, T. A. \& Gleason, J. L. Density functional theory guided design of exo-selective dehydroalanine dienophiles for application toward the synthesis of palau'amine. J. Org. Chem. 73, 102-110 (2008).

24. Hudon, J., Cernak, T. A., Ashenhurst, J. A. \& Gleason, J. L. Stable 5-substituted cyclopentadienes for the diels-alder cycloaddition and their application to the synthesis of palau'amine. Angew. Chem. Int. Ed. 47, 8885-8888 (2008).

25. Wang, S. \& Romo, D. Enantioselective synthesis of $(+)$-monobromophakellin and $(+)$-phakellin: a concise phakellin annulation strategy applicable to palau'amine. Angew. Chem. Int. Ed. 47, 1284-1286 (2008).

26. Li, Q. et al. Exploring symmetry-based logic for a synthesis of palau'amine. J. Org. Chem. 74, 5909-5919 (2009).

27. Sivappa, R., Mukherjee, S., Dias, H. V. R. \& Lovely, C. J. Studies toward the total synthesis of the oroidin dimers. Org. Biomol. Chem. 7, 3215-3218 (2009).

28. Namba, K., Inai, M., Sundermeier, U., Greshock, T. J. \& Williams, R. M. Synthetic studies on palau'amine. Construction of the cyclopentane core via an asymmetric 1,3-dipolar cycloaddition. Tetrahedron Lett. 51, 6557-6559 (2010).

29. Feldman, K. S., Nuriye, A. Y. \& Li, J. Extending pummerer reaction chemistry: studies in the palau'amine synthesis area. J. Org. Chem. 76, 5042-5060 (2011).

30. Ma, Z., Lu, J., Wang, X. \& Chen, C. Revisiting the kinnel-scheuer hypothesis for the biosynthesis of palau'amine. Chem. Commun. 47, 427-429 (2011).

31. Starr, J. T., Koch, G. \& Carreira, E. M. Enantioselective synthesis of the cyclopentyl core of the axinellamines. J. Am. Chem. Soc 122, 8793-8794 (2000).

32. O’Malley, D. P., Yamaguchi, J., Young, I. S., Seiple, I. B. \& Baran, P. S. Total synthesis of ( \pm )-axinellamines A and B. Angew. Chem. Int. Ed. 47, 3581-3583 (2008).

33. Su, S., Seiple, I. B., Young, I. S. \& Baran, P. S. Total syntheses of ( \pm )-massadine and massadine chloride. J. Am. Chem. Soc. 130, 16490-16491 (2008).

34. Su, S., Rodriguez, R. A. \& Baran, P. S. Scalable, stereocontrolled total synthesis of ( \pm )-axinellamines A and B. J. Am. Chem. Soc. 133, 13922-13925 (2011).

35. Ding, H., Roberts, A. G. \& Harran, P. G. Synthetic ( \pm )-axinellamines deficient in halogen. Angew. Chem. Int. Ed. 51, 4340-4343 (2012).

36. Ma, Z. et al. Asymmetric syntheses of sceptrin and massadine and evidence for biosynthetic enantiodivergence. Science 346, 219-224 (2014).

37. Wang, X. \& Chen, C. An approach for the synthesis of nakamuric acid. Tetrahedron 71, 3690-3693 (2015).

38. Ma, Y., De, S. \& Chen, C. Syntheses of cyclic guanidine-containing natural products. Tetrahedron 71, 1145-1173 (2015).

39. Wang, X. et al. Dimeric pyrrole-imidazole alkaloids: synthetic approaches and biosynthetic hypotheses. Chem. Commun. 50, 8628-8639 (2014).

40. Moris, J. C. \& Phillips, A. J. Marine natural products: synthetic aspects. Nat. Prod. Rep. 28, 269-289 (2011).

41. Gaich, T. \& Baran, P. S. Aiming for the ideal synthesis. Aiming for the ideal synthesis. J. Org. Chem. 75, 4657-4673 (2010).

42. Köck, M., Grube, A., Seiple, I. B. \& Baran, P. S. The pursuit of palau'amine. Angew. Chem. Int. Ed. 46, 6586-6594 (2007).

43. Jacquot, D. E. N. \& Lindel, T. Challenge palau'amine: current standings. Curr. Org. Chem. 9, 1551-1565 (2005).

44. Seiple, I. B. et al. Total synthesis of palau'amine. Angew. Chem. Int. Ed. 49, 1095-1098 (2010)

45. Seiple, I. B. et al. Enantioselective total synthesis of (-)-palau'amine, (-)-axinellamines, and (-)-massadines. J. Am. Chem. Soc. 133, 14710-14726 (2011).

46. Magnus, P., Garlzt, N., Selbert, K. A. \& Ornholt, A. Synthesis of carbamates from diethoxycarbonyl hydrazine derivatives by $\mathrm{E} 1 \mathrm{cB}$ eliminative cleavage of the N-N'-bond rather than reduction. Org. Lett. 11, 5646-5648 (2009).

47. Namba, K. et al. Toward palau'amine: $\mathrm{Hg}(\mathrm{OTf})_{2}$-catalyzed synthesis of the cyclopentane core. Chem. Eur. J. 15, 6560-6563 (2009).

48. Nishizawa, M., Imagawa, H. \& Yamamoto, H. Org. Biomol. Chem. 8, 511-521 (2010).

49. Namba, K., Shoji, I., Nishizawa, M. \& Tanino, K. 4-Aminopyridine catalyzed direct and regioselective acylation of $\mathrm{N}$-tosylhydrazide. Org. Lett. 11, 4970-4973 (2009).

50. Sakaitani, M. \& Ohfune, Y. Selective transformation of N-t-butoxycarbonyl group into n-alkoxy-carbonyl group via $\mathrm{n}$-carboxylate ion equivalent. Tetrahedron Lett. 26, 5543-5546 (1985).
51. Imaoka, T., Akimoto, T., Iwamoto, O. \& Nagasawa, K. Total synthesis of $(+)$-dibromophakellin and (+)-dibromophakellstatin. Chem. Asian J. 5, 1810-1816 (2010).

52. Shinada, T., Umezawa, T., Ando, T., Kozuma, H. \& Ohfune, Y. A new entry for the synthesis of $N$-acyl- $N$-substituted guanidines. Tetrahedron Lett. 47, 1945-1947 (2006).

53. Donohoe, T. J. et al. Direct dihydroxylation of cyclic allylic alcohols and trichloroacetamides using $\mathrm{OsO}_{4}$ /TMEDA. J. Org. Chem. 67, 7946-7956 (2002)

54. Grube, A., Immel, S., Baran, P. S. \& Köck, M. Angew. Chem. Int. Ed. 46, 6721-6724 (2007).

55. Shimizu, T., Hiranuma, S. \& Nakata, T. Efficient method for inversion of secondary alcohol by reaction of chloromethanesulfonates with cesium acetate Tetrahedron Lett. 37, 6145-6148 (1996).

56. Granelli-Pipemo, A. \& Nolan, P. Nuclear transcription factors that bind to elements of IL-2 promoter. Induction requirements in primary human T cells J. Immunol. 147, 2734-2739 (1991).

57. Steffan, N. M. et al. Regulation of IkB alpha phosphorylation by PKC- and $\mathrm{Ca}^{2+}$-dependent signal transduction pathways. J. Immunol. 155, 4685-4691 (1995).

58. Frisch, M. J. et al.Gaussian 09, revision D.01, Gaussian, Inc.,Wallingford, CT, (2009).

\section{Acknowledgements}

We thank Professor R.M. Williams (Colorado State University) for valuable discussion, Drs M. Tanaka, I. Okamoto and H. Imagawa (Tokushima Bunri University) for the $600 \mathrm{MHz}$ NMR and high-resolution mass spectra measurements of synthetic palau'amine, and Dr G. Hirai (RIKEN) for calculating the $\mathrm{pKa}$ values of synthetic intermediates. This work was partially supported by Grants-in-Aid for Scientific Research (numbers 24310162 and 26560449) and by a Grant-in-Aid for Scientific Research on Innovative Areas (Project No. 2301: Chemical Biology of Natural Products) from the Ministry of Education, Culture, Sports, Science and Technology, Japan. K.N. is grateful to the Naito Foundation, the Yamada Foundation and the Kurata Foundation for support through a Research Fund for Recently Independent Professors, and to Astellas Pharma Inc. and L'avenir 3000 Inc. for research funds. K. Takeuchi is grateful to JSPS for a Research Fellowship (no. 201502030) for Young Scientists. A portion of the density functional theory calculations were performed on supercomputers at the Research Center for Computational Science, Okazaki, Japan. Dedicated to late Professor Mugio Nishizawa.

\section{Author contribution}

K.N. and K. Tanino conceived the experiments and analysed the results. K. Takeuchi and Y.K. performed the laboratory experiments and optimized the reaction conditions, and Atsushi Nakayama helped their experiments. M.O. performed a biological test of immunosuppressive activity. Akira Nakayama performed the density functional theory calculations of key cascade reactions. M.Y. performed an X-ray diffraction study of 21 . K.N. wrote the paper and K. Tanino proofread the paper.

\section{Additional information}

Accession code: The X-ray crystallographic coordinates for structures 21 reported in this study have been deposited at the Cambridge Crystallographic Data Centre (CCDC), under deposition number 1417980. These data can be obtained free of charge from The Cambridge Crystallographic Data Centre via www.ccdc.cam.ac.uk/data_request/cif.

Supplementary Information accompanies this paper at http://www.nature.com/ naturecommunication

Competing financial interests: The authors declare no competing financial interests

Reprints and permission information is available online at http://npg.nature.com/ reprintsandpermissions/

How to cite this article: Namba, K. et al. Total synthesis of palau'amine. Nat. Commun 6:8731 doi: 10.1038/ncomms9731 (2015).

This work is licensed under a Creative Commons Attribution 4.0 International License. The images or other third party material in this article are included in the article's Creative Commons license, unless indicated otherwise in the credit line; if the material is not included under the Creative Commons license, users will need to obtain permission from the license holder to reproduce the material To view a copy of this license, visit http://creativecommons.org/licenses/by/4.0/ 